\title{
Efectividad y seguridad del tratamiento anticoagulante con acenocumarol en fibrilación auricular no valvular
}

\author{
CONSTANZA CASTRO ${ }^{1,2, a}$, LUIS BUSTOS ${ }^{1,4, a}$, \\ RENATO OCAMPO ${ }^{1,2, a}$, ELDA MOLINA ${ }^{3, \mathrm{~b}}$, PÍA CABRERO $^{4, \mathrm{~b}}$, \\ RENÉ VERGARA ${ }^{5}$, FERNANDO LANAS ${ }^{1,3, a}$
}

\section{Effectiveness and safety of oral anticoagulation treatment with acenocoumarol in non-valvular atrial fibrillation}

Background: Vitamin K antagonists significantly decrease the incidence of stroke but increase the risk of bleeding. Aim: To assess the effectiveness and risk of bleeding of vitamin Kantagonists in non-valvular atrial fibrillation. Material and Methods: Retrospective cohort study of 524 patients, 236 women (45\%) and 288 men (55\%) with non-valvular atrial fibrillation (NVAF) admitted to the oral anticoagulation treatment (OAT) clinic at four public hospitals, between 2009 and 2012. They were followed until March 2013, measuring the quality of OAT, ischemic and bleeding events. Results: The mean follow-up was 26.1 months, with 1,154.7 person-years of follow-up accrued. The percentage of time in therapeutic range (TTR) was $35.2 \pm 18 \%$; this was deemed to represent the quality of OAT. The cumulative incidence of ischemic events, either stroke or systemic embolism, was 2.25/100 person-years, being greater in patients with previous embolism (Risk ratio 5.21, 95\% confidence intervals 2.31- 11.73, $p<0.01$ ). The cumulative incidence of major bleeding events -extracranial and intracraneal-was 4.08/100 person-years. The main site of extracranial major bleeding was the gastrointestinal tract (32\%). Conclusions: In our clinical practice, the effectiveness of OAT with acenocoumarol in NVAF patients is similar to that published abroad. However, the incidence of bleeding complications is higher. The quality of the OAT measured by the TTR was lower than abroad.

(Rev Med Chile 2017; 145: 1243-1251)

Key words: Acenocoumarol; Anticoagulants; Atrial Fibrillation; Hemorrhage; Stroke.

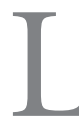

a fibrilación auricular (FA) es la arritmia sostenida más frecuente en adultos, afectando entre 1 y $2 \%$ de la población general ${ }^{1}$. La prevalencia aumenta con la edad, desde $0,7 \%$ entre los 55 y 59 años, hasta $17 \%$ en mayores de 85 años $^{2}$. En Chile no existe información sobre la prevalencia poblacional de FA, sin embargo, se asume -por el número de consultas y hospitalizaciones- que su prevalencia es similar a los estudios internacionales $^{3,4}$. La fibrilación auricular no valvular

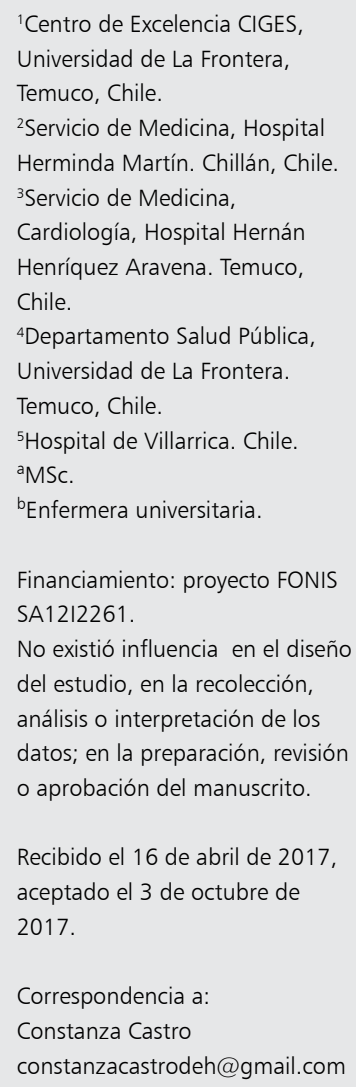

(FAVN) es un factor de riesgo independiente para el accidente cerebrovascular (ACV) isquémico, presentando un riesgo cinco veces mayor al de los pacientes que no tienen FANV ${ }^{5}$. Además, los ACV isquémicos de origen cardioembólico suelen ser más graves y tienen una tasa de supervivencia más baja que otros accidentes cerebrovasculares isquémicos ${ }^{6}$.

Los antagonistas de vitamina $\mathrm{K}(\mathrm{AVK})$-al compararse con el uso de placebo o de aspirina- 
han demostrado disminuir significativamente la incidencia de ACV isquémicos, ACV fatales o discapacitantes y mortalidad; manteniendo similares tasas de hemorragias intracraneales y extracraneales que la aspirina ${ }^{7,8}$. La calidad del tratamiento anticoagulante oral (TACO) se relaciona estrechamente no sólo a la incidencia de complicaciones embólicas y hemorrágicas, si no también con la gravedad letalidad de éstas9. El TACO tiene una estrecha ventana terapéutica y múltiples interacciones, por lo que es necesaria la monitorización frecuente con relación normalizada internacional (INR) para mantener a los pacientes dentro del rango terapéutico. En los ensayos clínicos controlados, los pacientes con anticoagulantes orales se mantienen con INR adecuado alrededor de 60-65\% del tiempo, sin embargo, en estudios del "mundo real" este porcentaje puede descender a menos de $50 \%$ del tiempo ${ }^{10-14}$.

El objetivo del estudio es evaluar la efectividad y seguridad del TACO con AVK que se realiza en hospitales públicos chilenos.

\section{Materiales y Método}

Se realizó un estudio de cohorte retrospectivo de pacientes con FANV que se hayan controlado en el policlínico de TACO de cuatro centros hospitalarios públicos de diferente complejidad. Los hospitales participantes fueron el Hospital Hernán Henríquez Aravena (Temuco, hospital tipo 1), el Hospital Herminda Martín (Chillán, hospital tipo 1), el Hospital de Villarrica (Villarrica, tipo 2) y Hospital de San Carlos (San Carlos, tipo 2).

\section{Cálculo de tamaño de muestra}

El tamaño de muestra se calculó en base a la incidencia de ACV isquémico ${ }^{15}$, un intervalo de confianza de $95 \%$, precisión absoluta de 1,25\% y una población estimada 260.000 personas con FA en el país ( $2 \%$ población general $)^{15}$. Se utilizó el programa EPIDAT versión 3.1, dando como resultado 505 años persona.

\section{Participantes}

Se evaluaron a todos los pacientes con diagnóstico de FANV o aleteo auricular que ingresaron por primera vez al policlínico de TACO de los hospitales referidos desde el año 2009 hasta el 31 de marzo del 2012. El seguimiento finalizó el 31 de marzo del 2013 o en la fecha de deceso.

\section{Criterios de inclusión}

Se incluyeron pacientes mayores de 18 años con diagnóstico de FANV o aleteo auricular no valvular, en tratamiento con acenocumarol, sin contraindicación de TACO y con riesgo de embolización moderado o alto $\left(\mathrm{CHADS}_{2} \geq 1\right)^{10}$.

\section{Criterios de exclusión}

Estenosis mitral moderada o severa, prótesis valvular mecánica o enfermedad tromboembólica venosa, participación en estudios clínicos de algún otro anticoagulante oral , cambio de policlínico de TACO (por ejemplo, debido a cambio de ciudad) $y$ haber iniciado el TACO en otro centro al menos tres meses previo al ingreso al policlínico de los hospitales señalados.

Se recolectaron datos de las fichas clínicas, registros hospitalarios y tarjetas TACO. Se entrevistó a los pacientes o familiares directos para complementar la información, especialmente eventos no registrados en la ficha clínica del hospital. En caso de abandonos, se contactó telefónicamente a los pacientes.

Se registraron características demográficas, tipo de arritmia, puntaje $\mathrm{CHA}_{2} \mathrm{DS}_{2}-\mathrm{VASc}$, puntaje HAS-BLED, y presencia de otros diagnósticos relevantes al momento de ingreso al programa ${ }^{16,17}$. Los valores de creatininemia y peso fueron obtenidos de la ficha clínica, en caso de no existir registro al ingreso, se utilizaron valores de creatininemia y peso hasta un año antes y después del ingreso. Se cuantificó el número de controles y calidad del TACO durante el seguimiento de los pacientes, para lo cual fueron excluidos los que tuvieron menos de 3 meses de control en el policlínico. La calidad del TACO se midió a través del tiempo dentro del rango terapéutico (TRT) usando el método de Rosendaal, incluyendo las interrupciones $^{18}$.

Se determinó la incidencia de ACV isquémico, embolización periférica, ACV hemorrágico, hemorragia intracraneal (HIC), hemorragia mayor, infarto agudo al miocardio y hemorragia no mayor clínicamente significativa, utilizándose las definiciones del estudio ARISTOTLE ${ }^{19}$. El ACV isquémico y embolías sistémicas se analizaron según intención de tratamiento, pero para las complicaciones hemorrágicas, se incluyeron sólo los eventos que se produjeron estando activos en TACO en el momento del suceso. En el caso de los pacientes que fallecieron durante el seguimiento, 
se revisó la ficha clínica y el certificado de defunción del Registro Civil. Se registró la suspensión de tratamiento, la cual podía ser por indicación médica de alta del policlínico TACO, o por abandono. Se definió como la inasistencia mayor a 60 días desde el término de la última prescripción médica ${ }^{20}$.

El estudio fue presentado y aprobado por los comités de ética correspondientes (Servicio de Salud Araucanía Sur y Hospital Herminda Martín). A los pacientes que se encontraban activos en el hospital, se solicitó consentimiento informado para el uso de la ficha clínica. Todos los datos fueron codificados para asegurar la confidencialidad de los participantes, conociendo la identidad de éstos sólo los encuestadores.

\section{Resultados}

Se identificaron 169 pacientes del Hospital Hernán Henríquez Aravena, 248 pacientes del Hospital Herminda Martín, 106 pacientes del Hospital San Carlos y 6 del Hospital de Villarrica. Se excluyeron tres pacientes del Hospital Herminda Martín porque sus fichas estaban extraviadas o incompletas, y dos pacientes del Hospital de San Carlos, quienes rechazaron participar. La cohorte incluyó 524 pacientes, 236 mujeres (45\%) y 288 hombres $(55 \%)$. El promedio de edad de ingreso al programa de TACO fue de 72,9 años $( \pm 9,65)$. Las características demográficas y clínicas de los pacientes al ingreso al programa están descritas en la Tabla 1. El promedio de puntaje $\mathrm{CHA}_{2} \mathrm{DS}_{2}-$ VASc al ingreso al programa fue 4,13 $\pm 1,52 \mathrm{y}$ la distribución de la cohorte según el puntaje $\mathrm{CHA}_{2} \mathrm{DS}_{2}$-VASc está presentada en la Figura 1. El promedio de puntaje HAS-BLED al ingreso al programa fue $2,7 \pm 0,88$, y la distribución de la cohorte según puntaje HAS-BLED está presentada en la Figura 2. La distribución porcentual de los pacientes según clearence de creatinina calculado al momento de ingreso al programa se presenta en la Figura 3. Otros diagnósticos relevantes que se registraron al momento del ingreso fueron insuficiencia renal crónica $(8,2 \%)$, daño hepático crónico $(2,9 \%)$, dislipidemia $(20,1 \%)$, antecedente de hemorragia digestiva $(3,8 \%)$, úlcera digestiva $(1,2 \%)$, gastritis $(5,2 \%)$, y enfermedad pulmonar obstructiva crónica $(9,4 \%)$. El 15,6\% de los pacientes utilizaban algún antiagregante plaquetario y $34 \%$ un inhibidor de secreción gástrica.
Tabla 1. Características demográficas y clínicas de los pacientes al momento de ingreso al programa TACO

\begin{tabular}{|c|c|}
\hline Variable & Resultado \\
\hline Edad al ingreso (años) & Promedio, DE \\
\hline Mujeres & $74 \pm 8,7$ \\
\hline Hombres & $71 \pm 10,19$ \\
\hline Total & $72,9 \pm 9,65$ \\
\hline Tipo de arritmia al ingreso & n $\quad(\%)$ \\
\hline FA permanente & $373(71,9 \%)$ \\
\hline FA paroxística & $120(23,1 \%)$ \\
\hline Flutter auricular & $26(5 \%)$ \\
\hline Sin diagnóstico & $5(1 \%)$ \\
\hline $\begin{array}{l}\text { Presencia de factores de riesgo } \\
\text { embolización }\end{array}$ & n $\quad(\%)$ \\
\hline Insuficiencia cardíaca & $235(44,9 \%)$ \\
\hline HTA & $487(92,9 \%)$ \\
\hline Edad $\geq 75$ años & $245(46,8 \%)$ \\
\hline DM & $154(29,4 \%)$ \\
\hline Antecedentes de TIA o ACV isquémico & $158(30,2 \%)$ \\
\hline Antecedente de enfermedad vascular & $61(11,6 \%)$ \\
\hline Edad entre 65 y 74 años & $178(34 \%)$ \\
\hline Sexo femenino & $236(45 \%)$ \\
\hline Riesgo de embolización & Promedio, DE \\
\hline $\mathrm{CHADS}_{2}$ & $2,73 \pm 1,16$ \\
\hline $\mathrm{CHA}_{2} \mathrm{DS}_{2}-\mathrm{VASC}$ & $4,12 \pm 1,52$ \\
\hline \multicolumn{2}{|l|}{ Riesgo de hemorragia } \\
\hline HAS-BLED & $2,70 \pm 0,88$ \\
\hline Función renal & Promedio, DE \\
\hline Creatinina $\mathrm{mg} / \mathrm{dl}^{*}$ & $1,09 \pm 0,74$ \\
\hline Peso kg** & $73,6 \pm 15,5$ \\
\hline $\begin{array}{l}\text { Clearence de creatinina según } \\
\text { Cockcroft-Gault } \mathrm{ml} / \mathrm{min}^{* * *}\end{array}$ & $68,7 \pm 28,3$ \\
\hline
\end{tabular}

HTA: Hipertensión arterial; DM: Diabetes mellitus; TIA: Accidentes vascular transitorio; ACV: accidente cerebrovascular; $\mathrm{CHADS}_{2}$ y $\mathrm{CHA}_{2} \mathrm{DS}_{2}$-VASc: acrónimos de escalas de riesgo de embolización para pacientes con fibrilación auricular no valvular. HAS-BLED: acrónimo de escala de riesgo de hemorragia en pacientes con fibrilación auricular no valvular en tratamiento anticoagulante. ${ }^{*}$ Datos de 499 pacientes. **Datos de 333 pacientes. ${ }^{* *}$ Datos de 325 pacientes.

El tiempo de seguimiento de la cohorte promedió $26,4 \pm 11,2$ meses, con un seguimiento mínimo de un día (paciente que falleció al día siguiente de inicio TACO) y un seguimiento máximo de 51,8 meses, completando un total de $1.154,7$ años persona. Durante el seguimiento, 110 pacientes $(20,9 \%)$ suspendieron el programa 

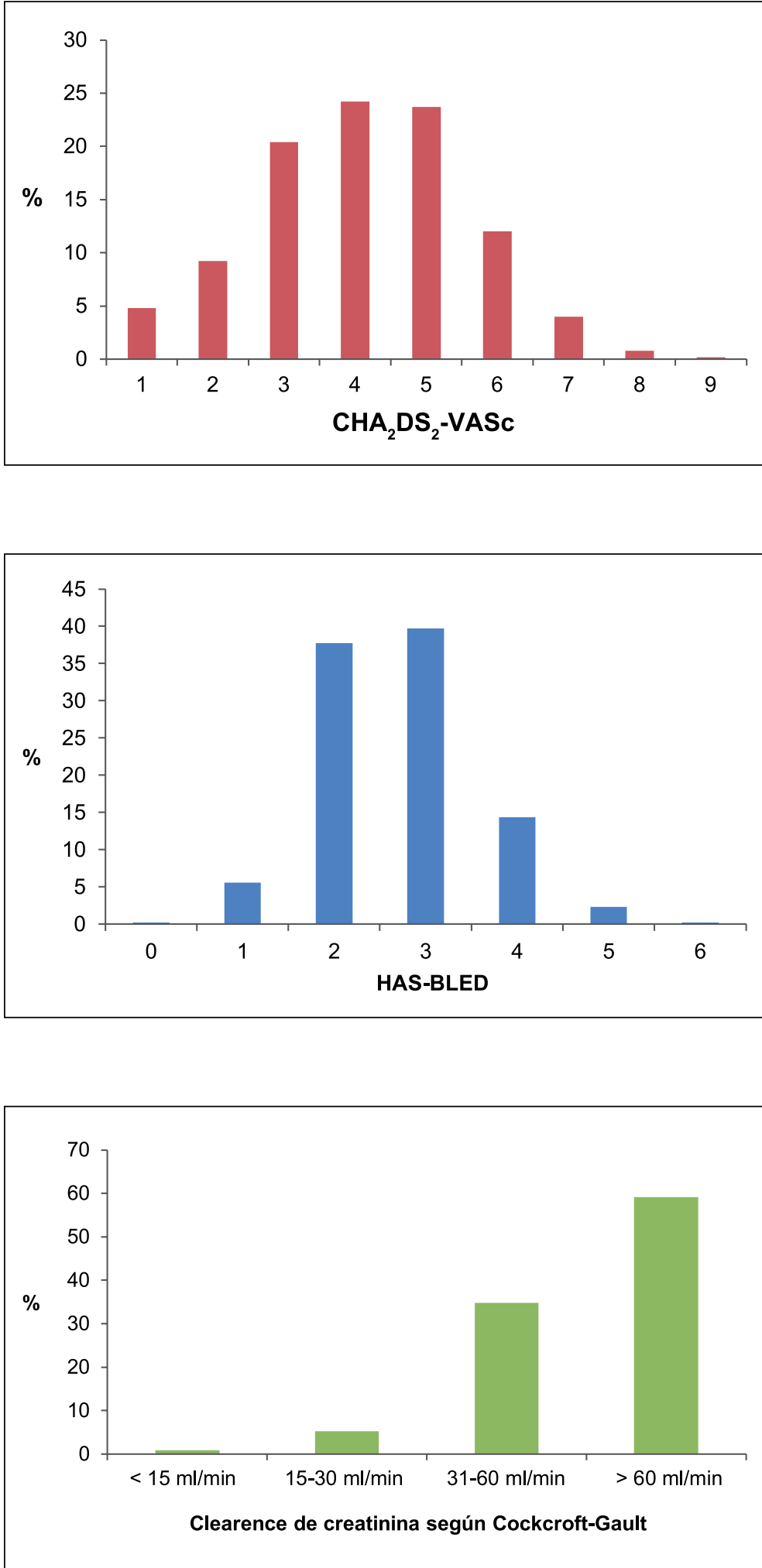

Figura 1. Distribución porcentual de pacientes según $\mathrm{CHA}_{2} \mathrm{DS}_{2}$-VASc al ingreso al programa. $\mathrm{CHA}_{2} \mathrm{DS}_{2}$-VASc: acrónimo de escala de riesgo. (Insuficiencia cardíaca congestiva o fracción de eyección ventrículo izquierdo $<40 \%$, hipertensión arterial, edad mayor a 75 años, diabetes, antecedentes de accidente vascular isquémico o accidente vascular transitorio, enfermedad vascular, edad $>65$ y sexo femenino).

Figura 2. Distribución porcentual de pacientes según HAS-BLED al ingreso al programa. HAS-BLED: acrónimo de escala de riesgo de hemorragia en pacientes con FA no valvular. (Hipertensión arterial, función hepática o renal alterada, accidente cerebrovascular previo, historia de sangrado, INR lábil, edad > 65 años, consumo de fármacos que aumentan riesgo de sangrado y consumo de alcohol).

Figura 3. Distribución de pacientes (datos de 325 pacientes). según rangos de clearence de creatinina 
al menos en una ocasión; de estos, 35,5\% lo hizo por indicación médica. De los 71 pacientes que abandonaron, $42,3 \%$ retornó al programa. La duración promedio del abandono fue 7,7 meses. Las principales causas identificadas de abandono de tratamiento fueron dificultades para el traslado al control (22\%), dificultades para conseguir horas $(15 \%)$ y falta de red de apoyo (12\%). Solo en dos pacientes en abandono no se logró completar su seguimiento. Se verificó su estado vital a través del Servicio de Registro Civil e Identificación y se revisó que no hubieran tenido eventos a través de sus consultorios y registros hospitalarios de su provincia. Dado estos antecedentes, se incorporaron al análisis como abandono, sin eventos.
El tiempo que la cohorte se mantuvo en TACO promedió 23,6 \pm 12,1 meses, con seguimiento mínimo de un día (paciente que falleció al día siguiente de inicio TACO) y seguimiento máximo de 51,8 meses, completando un total de 1.029,8 años persona. La dosis promedio semanal de acenocumarol fue $12,2 \pm 5,9 \mathrm{mg}$ con un rango entre 3 a $46 \mathrm{mg}$. El promedio de controles mensuales fue $1 \pm 0,2$, con un rango de 0,3 a 2,7. La calidad de la anticoagulación de los pacientes de la cohorte medida a través del TRT promedió $35,2 \pm 18 \%$ y el porcentaje de test dentro del rango fue $29,6 \%$. Sólo el 8,2 \% de los pacientes alcanzaron un TRT $\geq 60 \%$ (Figura 4 ).

La incidencia de eventos embólicos, ya sea

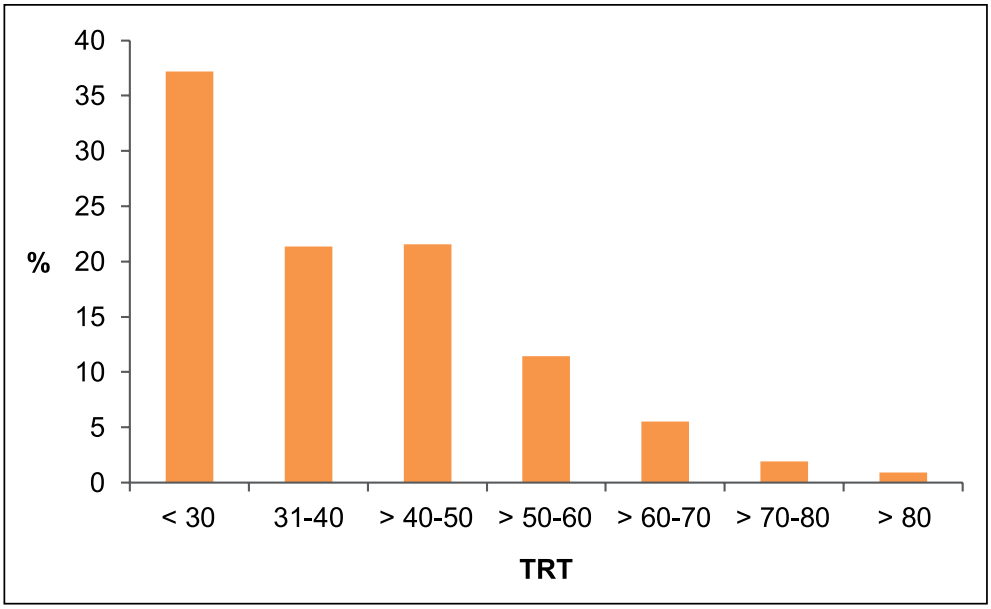

Figura 4. Distribución porcentual de pacientes según rangos de tiempo dentro del rango terapéutico. TRT: porcentaje del tiempo en rango terapéutico. * Se excluyeron los pacientes que tenían menos de tres meses de $\mathrm{TACO}^{26}$.

Tabla 2. Frecuencia e incidencia acumulada de accidente vascular cerebral isquémico y embolizaciones sistémicas

\begin{tabular}{|lcc|}
\hline Evento & $\mathbf{n}$ & Incidencia acumulada \\
\hline ACV isquémico leve & 7 & $0,61 / 100$ pacientes año \\
\hline ACV isquémico moderado & 4 & $0,35 / 100$ pacientes año \\
\hline ACV isquémico severo & 4 & $0,35 / 100$ pacientes año \\
\hline ACV isquémico fatal & 7 & $0,61 / 100$ pacientes año \\
\hline ACV isquémico total & 22 & $1,91 / 100$ pacientes año \\
\hline Embolización sistémica no fatal & 2 & $0,17 / 100$ pacientes año \\
\hline Embolización sistémica fatal & 2 & $0,17 / 100$ pacientes año \\
\hline Embolización sistémica total & 4 & $0,35 / 100$ pacientes año \\
\hline Total eventos embólicos & 26 & $2,25 / 100$ pacientes año \\
\hline
\end{tabular}

ACV: accidente cerebrovascular. 
ACV isquémico o embolización sistémica, fue de 2,25/100 pacientes año (Tabla 2). La letalidad al mes del evento ACV isquémico fue $31,8 \%$ y de embolización sistémica fue $50 \%$. Ningún paciente presentó dos veces el mismo evento durante el seguimiento.

El análisis de asociación de distintas variables con eventos embólicos se muestra en la Tabla 3. Los pacientes que presentaron eventos embólicos tenían mayor puntaje $\mathrm{CHA}_{2} \mathrm{DS}_{2}-\mathrm{VASc}(5,0$ vs $4,0$, t test $\mathrm{p}<0,00)$, TRT menor $(25,4$ vs $35,7 \%$, $\mathrm{p}<0,01$ ), sin presentar diferencia en la frecuencia de controles ni dosis de acenocumarol.

En relación a los eventos embólicos fatales, los pacientes con antecedentes de embolización, diabetes mellitus (DM) y enfermedad vascular tu-

Tabla 3. Asociación de variables con eventos embólicos total

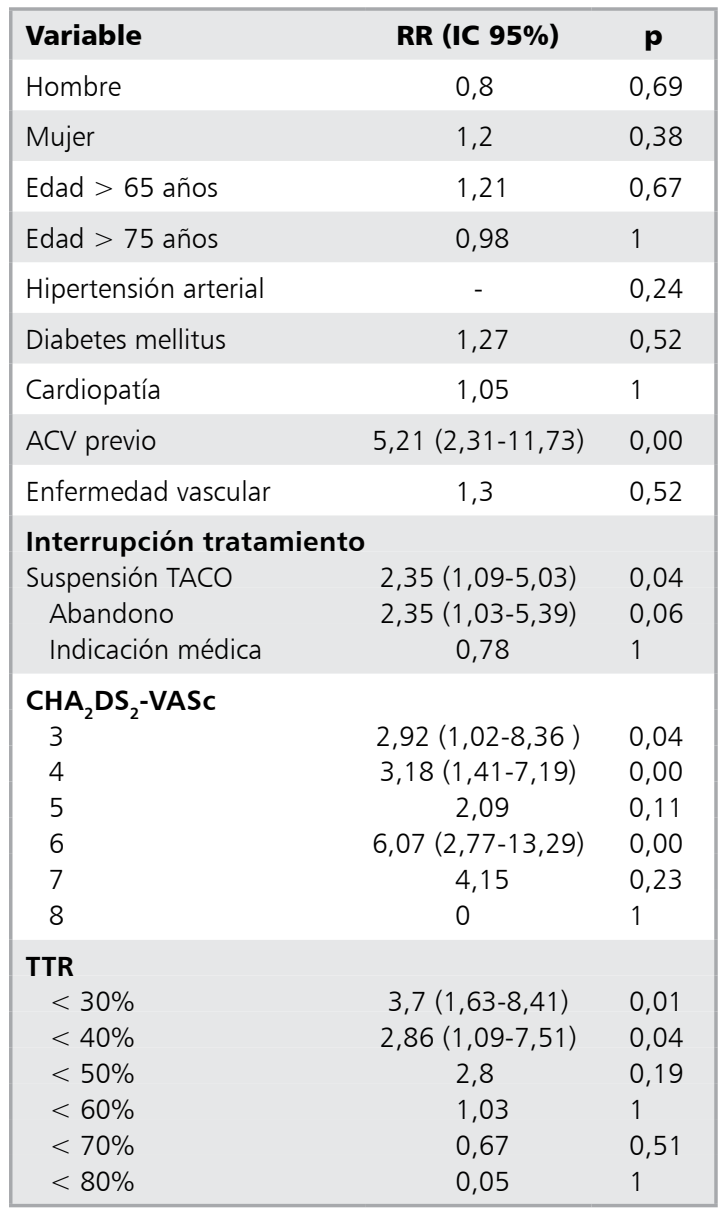

vieron significativamente más eventos embólicos ( RR 8,11 p < 0,004; RR 4,9 p < 0,02; RR 6,07 p 0,01 ; respectivamente).

La incidencia de eventos hemorrágicos mayores extracraneales e intracraneales (ACV hemorrágicos, HIC y otras hemorragias mayores) fue de 4,08/100 pacientes año (Tabla 4). El sitio de sangrado de las otras hemorragias mayores más frecuente fue el gastrointestinal (32\%). Otro evento medido fue la hemorragia no mayor clínicamente relevante (HNMCR), con 33 eventos (3,24 /100 pacientes año).

No se encontraron asociaciones con eventos hemorrágicos en el análisis crudo con las variables estudiadas excepto con TRT $<40 \%$.

La incidencia de infarto al miocardio no fatal fue $0,26 / 100$ pacientes año $(n=3)$ y de infarto fatal $0,53 / 100$ pacientes años $(n=6)$. La mortalidad por eventos embólicos fue $0,61 / 100$ pacientes año $y$ por eventos hemorrágicos fue $1,28 / 100$ pacientes año. La mortalidad general durante seguimiento fue de $8,43 / 100$ pacientes año $(\mathrm{n}=96)$, lo cual se asociaba a TRT $<50 \%$ (RR 2,77; IC95\% 1,32 $5,81 ; \mathrm{p} 0,03)$.

\section{Discusión}

Existen diversos estudios de cohorte sobre la efectividad y seguridad del uso de TACO en pacientes con FANV en la práctica clínica en Europa y Norteamérica ${ }^{21}$. Un análisis compartido de estos estudios realizado por Ogilvie estima una incidencia de embolizaciones y hemorragias mayores de 1,7 y 1,8 eventos/100 pacientes año, respectivamente ${ }^{21}$.

Estudios más recientes se basan en poblaciones de mayor riesgo. Burton publicó en 2006 una cohorte regional retrospectiva de pacientes con FANV persistente que se controlaban en atención primaria de una región de Escocia ${ }^{22}$. El promedio de edad era 77 años y $46 \%$ de los pacientes tenían antecedentes de embolización. La incidencia de eventos embólicos fue 2,2/100 pacientes año, similar a la obtenida en nuestra cohorte, $y$ la incidencia de hemorragias mayores fue menor (2,6/100 pacientes año). La calidad del TACO fue mayor a nuestra cohorte, con un TRT promedio de $68 \%$.

Poli, en 2009, publicó un estudio de cohorte prospectiva de pacientes con FANV en TACO que 
Tabla 4. Frecuencia e incidencia acumulada de los eventos hemorrágicos

\begin{tabular}{|lcc|}
\hline Evento & $\mathbf{n}$ & Incidencia \\
\hline ACV hemorrágico leve & 1 & $0,1 / 100$ pacientes año \\
\hline ACV hemorrágico moderado & 0 & $0 / 100$ pacientes año \\
\hline ACV hemorrágico severo & 0 & $0 / 100$ pacientes año \\
\hline ACV hemorrágico fatal & 8 & $0,78 / 100$ pacientes año \\
\hline ACV hemorrágico total & 9 & $0,87 / 100$ pacientes año \\
\hline HIC no fatal & 1 & $0,1 / 100$ pacientes año \\
\hline HIC fatal & 7 & $0,68 / 100$ pacientes año \\
\hline HIC total & 8 & $0,78 / 100$ pacientes año \\
\hline Hemorragia mayor no fatal & 20 & $1,94 / 100$ pacientes año \\
\hline Hemorragia mayor fatal & 5 & $0,49 / 100$ pacientes año \\
\hline Hemorragia mayor total & 25 & $2,42 / 100$ pacientes año \\
\hline Total eventos hemorrágicos & 42 & $4,08 / 100$ pacientes año \\
\hline
\end{tabular}

ACV: accidente cerebrovascular; HIC: hemorragia intracraneal.

Tabla 5. Asociación de variables con eventos hemorrágicos mayores

\begin{tabular}{|c|c|c|}
\hline Variable & RR (IC 95\%) & $\mathbf{p}$ \\
\hline Hombre & 0,82 & 0,59 \\
\hline Mujer & 1,35 & 0,38 \\
\hline Edad $>65$ años & 0,70 & 0,45 \\
\hline HTA & 2,51 & 0,49 \\
\hline Función renal alterada & 1,93 & 0,8 \\
\hline Función hepática alterada & 2,12 & 0,25 \\
\hline ACV previo & 1,43 & 0,33 \\
\hline Historia de sangrado & 1,32 & 0,37 \\
\hline INR lábil & 0,45 & 0,13 \\
\hline Medicación predisponente & 0,29 & 0,34 \\
\hline Alcoholismo & 1,4 & 0,64 \\
\hline \multicolumn{3}{|l|}{ HAS-BLED* } \\
\hline$>0$ & - & - \\
\hline$>1$ & 2,04 & 0,71 \\
\hline$>2$ & 0,98 & 1 \\
\hline$>3$ & 1,06 & 0,81 \\
\hline$>4$ & 0 & - \\
\hline$>5$ & 0 & - \\
\hline \multicolumn{3}{|l|}{ TTR } \\
\hline$<30 \%$ & $3,98(1,58-10,84)$ & 0,01 \\
\hline$<40 \%$ & $2,61(0,99-8,09)$ & 0,04 \\
\hline$<45 \%$ & 1,54 & 0,39 \\
\hline$>60 \%$ & 0,85 & 1 \\
\hline
\end{tabular}

* No hubo eventos hemorrágicos mayores en pacientes con HAS-BLED 0, 5 ni 6. se controlaban en una clínica universitaria de la Universidad de Florencia, Italia ${ }^{23}$. La población estudiada era también similar a nuestra cohorte: promedio de edad 75 años, 34\% antecedentes de embolización, pero con menos pacientes hipertensos (60\% versus $92 \%)$ y menos pacientes con insuficiencia cardíaca (21\% versus $44,9 \%)$. La incidencia de eventos embólicos fue menor a la observada en nuestra cohorte (1,67/ 100 pacientes año) y, además, hubo menos embolizaciones fatales $(5,5 \%)$ y la mayoría fueron leves (44\% Rankin modificado 1-2). La calidad de TACO fue similar a los otros estudios, con TRT promedio de $68 \%$.

El registro GARFIELD-AF es un registro multicéntrico que incluye a pacientes con diagnóstico reciente de FA. Recientemente fueron publicados los desenlaces a un año del inicio de TACO, informando una incidencia de embolizaciones y hemorragias mayores 1,13 y 1,27 eventos/100 pacientes año, respectivamente. Estos resultados son muy diferentes a los de nuestra cohorte, pero hay que considerar que el perfil de riesgo es menor (menor edad, $\mathrm{CHA}_{2} \mathrm{DS}_{2}$-VASc y HAS-BLED) ${ }^{24}$.

La calidad de anticoagulación en nuestra cohorte, medida a través del TRT, es marcadamente menor a lo publicado en la literatura ${ }^{25}$. Sin embargo, en la interpretación de este resultado debe considerarse que -a diferencia de muchos estudios- el TRT se calculó incluyendo los tiempos de interrupción con el objeto de representar mejor la realidad. 
En relación al estudio de asociación, la incidencia de los desenlaces es baja y el análisis realizado es univariado, por lo que los resultados deben ser interpretados con cautela. A pesar de ello, estos concuerdan con los factores y escalas de riesgo de embolización conocidas, asignándose mayor riesgo al antecedente de embolización, mayor puntaje $\mathrm{CHA}_{2} \mathrm{DS}_{2}$-VASc, menor calidad de TACO, y suspensión de TACO. En relación a esto último, este estudio revela la dificultad de nuestros pacientes para permanecer en TACO por razones de accesibilidad, ya sea por dificultades de trasporte, dificultad para obtener horas clínicas y falta de red de apoyo. Esto requiere un análisis multidisciplinario con el objeto de facilitar el acceso al tratamiento que ha probado ser eficaz.

La principal limitación de este estudio es que se basa en una cohorte retrospectiva, por lo que la calidad de la información es menor al compararla con estudios prospectivos. Para compensar esto, se utilizaron diversas fuentes de información, contactando prácticamente todos los casos vivos. En relación a esto mismo, se decidió no incluir como eventos las crisis isquémicas transitorias, cuyo diagnóstico es más impreciso. En las fortalezas del estudio debemos mencionar que, en nuestro conocimiento, no existe otro estudio sobre la efectividad y seguridad del TACO en FANV en Chile. El seguimiento fue muy alto, lo que fortalece la validez interna del estudio. Por último, al incluir centros de diversa complejidad y con población rural y urbana, creemos que es representativo de la realidad nacional en el sistema público chileno.

En resumen, si bien la efectividad del TACO en nuestro medio está dentro de los estándares internacionales, la alta incidencia y letalidad de los eventos hemorrágicos demuestran un déficit en la seguridad de este, lo cual pudiese ser explicado por la calidad de TACO.

Agradecimientos: Agradecemos a las personas que apoyaron este proyecto en los diferentes centros, como a Patricio Gajardo e Ivonne Navarrete del CIGES, a Juana Urra del Hospital de San Carlos, a Juana Cifuentes del Hospital Hernán Henríquez Aravena, y Florentina Llanos y Aurelia Coloma del Hospital Herminda Martín. Por último, agradecemos especialmente a Carlos Vallejos, quien apoyó en el diseño y análisis del proyecto.

\section{Referencias}

1. Go A, Hylek E, Phillips K, Chang Y, Henault L, Selby J, et al. Prevalence of diagnosed atrial fibrillation in adults: National implications for rhythm management and stroke prevention: The anticoagulation and risk factors in atrial fibrillation (ATRIA) study. JAMA 2001; 285: 2370-5.

2. Heeringa J, van der Kuip D, Hofman A, Kors J, van Herpen G, Stricker B, et al. Prevalence, incidence and lifetime risk of atrial fibrillation: The Rotterdam study. European Heart Journal 2006; 27: 949-53.

3. Ortiz M. Hospitalizaciones asociadas al diagnóstico de fibrilación auricular en Chile: Tendencia creciente en la última década. Rev Chil Cardiol 2011; 30: 193-7.

4. Santelices E. Programa ACG y GRD. Departamento de desarrollo estratégico. Ministerio de Salud Chile, 2013.

5. Wolf P, Abbott R, Kannel W. Atrial fibrillation as an independient risk factor for stroke: The Framinghan Study. Stroke 1991; 22: 983-8.

6. Kolominsky-Rabas P, Weber M, Gefeller O, Neundoerfer B, Heuschmann P. Epidemiology of ischemic stroke subtypes according to toast criteria: Incidence, recurrence, and long-term survival in ischemic stroke subtypes: A population-based study. Stroke 2001; 32: 2735-40.

7. Aguilar M, Hart R, Pearce L. Oral anticoagulants versus antiplatelet therapy for preventing stroke in patients with non-valvular atrial fibrillation and no history of stroke or transient ischemic attacks. The Cochrane database of systematic reviews. 2007: CD006186. Disponible en www.bvsalud.org [Consultado el 17 de abril de 2017].

8. Saxena R, Koudstaal P. Anticoagulants versus antiplatelet therapy for preventing stroke in patients with nonrheumatic atrial fibrillation and a history of stroke or transient ischemic attack. The Cochrane database of systematic reviews. 2004: CD000187. Disponible en www.bvsalud.org [Consultado el 17 de abril de 2017].

9. Hylek E, Go A, Chang Y, Jensvold N, Henault L, Selby J, et al. Effect of intensity of oral anticoagulation on stroke severity and mortality in atrial fibrillation. NEJM 2003; 349: 1019-26.

10. Camm A, Kirchhof P, Lip G, Schotten U, Savelieva I, Ernst $S$, et al. Guías de práctica clínica para el manejo de la fibrilación auricular. Rev Esp Cardiol 2011; 64: 439-40.

11. Ansell J, Hirsh J, Hylek E, Jacobson A, Crowther M, Palareti G. Pharmacology and management of the vitamin k antagonists: American college of chest physicians evidence-based clinical practice guidelines. Chest 2008; 133: 160S-98S.

12. Chan P, Li W, Hai J, Chan E, Wong I, Tse H, et al. Time 
in therapeutic range and percentage of international normalized ratio in the therapeutic range as a measure of quality of anticoagulation control in patients with atrial fibrillation. Canadian Journal of Cardiology 2016; 32 (10): 1247.e23-8.

13. Haas S, Ten Cate H, Accetta G, Angchaisuksiri P, Bassand JP, Camm AJ, et al. Quality of vitamin k antagonist control and 1-year outcomes in patients with atrial fibrillation: A global perspective from the GARFIELD-AF Registry. PloS One 2016; 11: e0164076. Disponible en www.pubmed.com [Consultado el 17 de abril de 2017].

14. Esposti D, Sangiorgi D, Di Pascale G, Gensini G, Iori I, Buda S, et al. Adherence to treatment and anticoagulation control in vitamin-k antagonists treated-patients: An administrative databases analysis in a large italian population. Farmeconomia 2011; 12: 63-9.

15. Hart R, Pearce L, Aguilar M. Meta-analysis: Antithrombotic therapy to prevent stroke in patients who have nonvalvular atrial fibrillation. Ann Intern Med 2007; 146: 857-67.

16. Lip G, Nieuwlaat R, Pisters R, Lane D, Crijns H. Refining clinical risk stratification for predicting stroke and thromboembolism in atrial fibrillation using a novel risk factor-based approach: The Euro Heart Survey on Atrial Fibrillation. Chest. 2010; 137: 263-72.

17. Lip G, Andreotti F, Fauchier L, Huber K, Hylek E, Knight $\mathrm{E}$, et al. Bleeding risk assessment and management in atrial fibrillation patients. Executive summary of a position document from the European Heart Rhythm Association [EHRA], endorsed by the European Society of Cardiology [ESC] working group on thrombosis.
Thromb Haemos 2011; 106: 997-1011.

18. Rosendaal F, Cannegieter S, van der Meer F, Briet E. A method to determine the optimal intensity of oral anticoagulant therapy. Thromb Haemos 1993; 69: 236-9.

19. Lopes R, Alexander J, Al-Khatib S, Ansell J, Díaz R, Easton JD, et al. Apixaban for reduction in stroke and other thromboembolic events in atrial fibrillation (ARISTOTLE) trial: Design and rationale. Am Heart J 2010; 159: 331-9.

20. Fang M. Warfarin discontinuation after starting warfarin for atrial fibrillation. Cardiovasc Qual Outcomes 2010; 3: 624-31.

21. Ogilvie I, Welner S, Cowell W, Lip G. Ischaemic stroke and bleeding rates in 'real-world' atrial fibrillation patients. Thromb Haemos 2011; 106: 34-44.

22. Burton C, Isles C, Norrie J, Hanson R, Grubb E. The safety and adequacy of antithrombotic therapy for atrial fibrillation: A regional cohort study. The British Journal of General Practice 2006; 56: 697-702.

23. Poli D, Antonucci E, Marcucci R, Mannini L, Falciani $\mathrm{M}$, Abbate R, et al. The quality of anticoagulation on functional outcome and mortality for tia/stroke in atrial fibrillation patients. International Journal of Cardiology 2009; 132: 109-13.

24. Camm AJ, Accetta G, Ambrosio G, Atar D, Bassand JP, Berge E, et al. Evolving antithrombotic treatment patterns for patients with newly diagnosed atrial fibrillation. Heart 2017; 103: 307-14.

25. Mearns E, White C, Kohn C, Hawthorne J, Song J, Meng $\mathrm{J}$, et al. Quality of vitamin k antagonist control and outcomes in atrial fibrillation patients: A meta-analysis and meta-regression. Thrombosis Journal 2014; 12: 14. 